(1)

CrossMark

\title{
Inhaled corticosteroids and COVID-19: a systematic review and clinical perspective
}

\author{
David M.G. Halpin ${ }^{1,2}$, Dave Singh ${ }^{2,3}$ and Ruth M. Hadfield ${ }^{2,4}$
}

Affiliations: 'University of Exeter Medical School, College of Medicine and Health, University of Exeter, Exeter, UK. ${ }^{2}$ Global Initiative for Chronic Obstructive Lung Disease (GOLD). ${ }^{3}$ University of Manchester, Manchester University NHS Foundation Trust, Manchester, UK. ${ }^{4}$ Macquarie University, Sydney, Australia.

Correspondence: David M.G. Halpin, University of Exeter Medical School, Smeall Building, St Luke's Campus, Magdalen Road, Exeter EX1 2LU, UK. E-mail: d.halpinanhs.net

@ERSpublications

There is no evidence on benefits or harms of inhaled steroids in COVID-19. It is essential that epidemiological studies of COVID-19 include detailed information on comorbidities and prior medication to help answer this question. https://bit.ly/2XVwIsa

Cite this article as: Halpin DMG, Singh D, Hadfield RM. Inhaled corticosteroids and COVID-19: a systematic review and clinical perspective. Eur Respir J 2020; 55: 2001009 [https://doi.org/10.1183/ 13993003.01009-2020].

The current coronavirus 2019 (COVID-19) pandemic, caused by severe acute respiratory syndrome-coronavirus 2 (SARS-CoV-2) infection, raises important questions as to whether pre-morbid use or continued administration of inhaled corticosteroids (ICS) affects the outcomes of acute respiratory infections due to coronavirus. Many physicians are concerned about whether individuals positive for SARS-CoV-2 and taking ICS should continue them or stop them, given that ICS are often regarded as immunosuppressive. A number of key questions arise. Are people with asthma or COPD at increased risk of developing COVID-19? Do ICS modify this risk, either increasing or decreasing it? Do ICS influence the clinical course of COVID-19? (figure 1). Whether ICS modify the risk of developing COVID-19 or the clinical course of COVID-19 in people who do not have lung disease should also be considered (figure 1).

COVID-19 has an initial period characterised by cough and fever, followed by the development of dyspnoea after around 8 days in $\sim 20 \%$ of patients, with pulmonary infiltrates in about $10 \%[1,2]$. Approximately $25 \%$ of patients admitted to hospital developed acute respiratory distress syndrome (ARDS) a median 10.5 days after symptom onset [2].

In vitro models suggest that there is impaired interferon production and other antiviral innate immune responses to experimental rhinovirus and influenza infection in both asthma and COPD, and this could potentially increase susceptibility to viral infections including COVID-19. However, not all studies have replicated these findings [3]. For instance, a study of the response of asthmatic children to natural colds, including some due to coronavirus, showed an appropriate innate response [4]. These contrasting results may reflect the heterogeneity in innate immune responses between individuals and/or variability in the response to different viruses [5].

Surprisingly, the prevalence of chronic respiratory disease among patients with SARS and COVID-19 appears to be lower than among the general population [6]. This is not the case for other chronic diseases and leads us to hypothesise that lung disease, patients' behaviour or, more likely, their treatment may have some protective effect. Sadly, patients with underlying lung disease who develop COVID-19 and are hospitalised have worse outcomes, with a case fatality rate of $6.3 \%$ compared to $2.3 \%$ overall in China [7].

Received: 03 April 2020 | Accepted after revision: 16 April 2020

Copyright OERS 2020. This version is distributed under the terms of the Creative Commons Attribution NonCommercial Licence 4.0. 


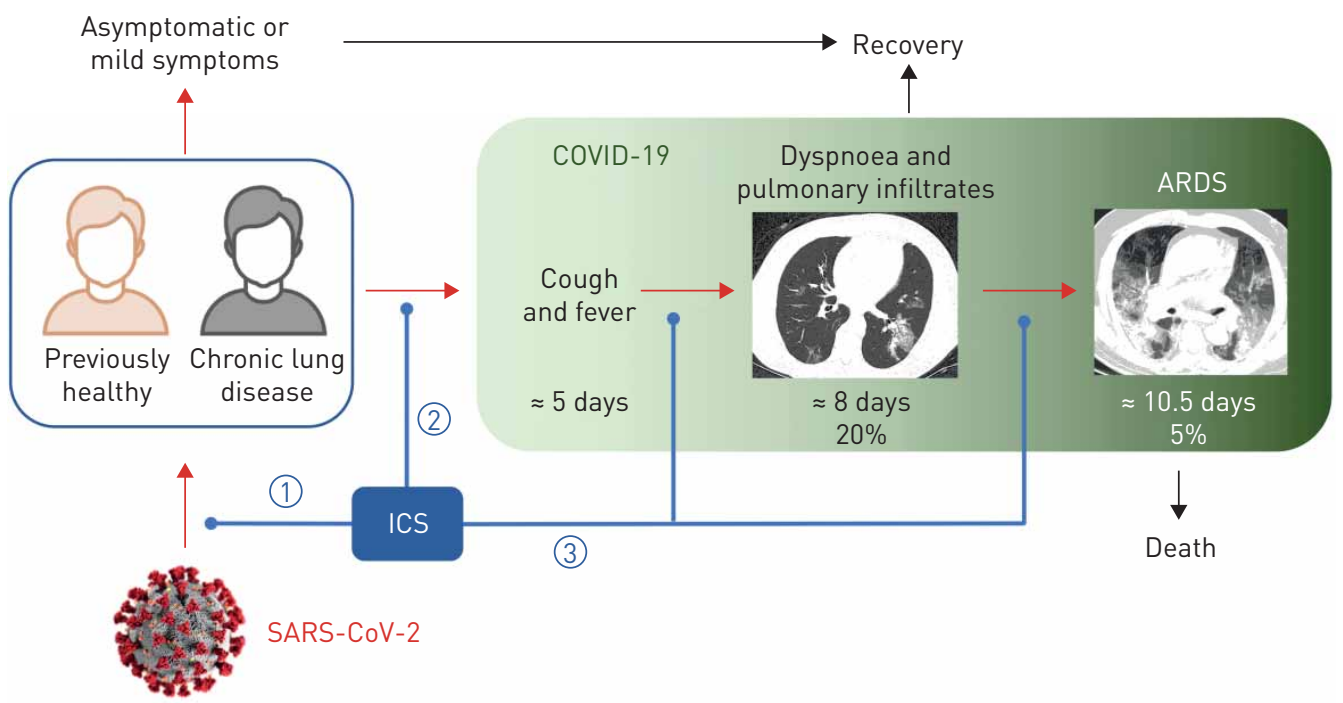

FIGURE 1 Potential steps in the development and evolution of severe acute respiratory syndrome-coronavirus 2 (SARS-CoV-2) infection that may be affected beneficially or adversely by inhaled corticosteroids (ICS). 1) Infection with SARS-CoV-2; 2) development of coronavirus 2019 (COVID-19) disease; 3) progression of COVID-19. ARDS: acute respiratory distress syndrome.

These individuals may have less reserve to cope with the pulmonary effects of severe infection or their immunopathological abnormalities may make them more susceptible to developing pulmonary inflammation and ARDS.

ICS, alone or in combination with bronchodilators, are used extensively in the treatment of asthma [8], and combined with bronchodilators have a role in the management of some patients with COPD [9]. There are a number of paradoxes about their effects on viral infections and exacerbation rates which are relevant when considering ICS use during the COVID-19 pandemic. ICS use in asthma and COPD is associated with an increased risk of upper respiratory tract infections $[10,11]$. In people with COPD, ICS use is associated with a higher prevalence of pneumonia [12] and a change in the lung microbiome, although not a change in respiratory virus detection [13]. The evidence in asthma is less clear cut, but at least one observational study has shown an increased risk of pneumonia or lower respiratory infection [14]. In vitro studies have suggested that corticosteroids may impair antiviral innate immune responses $[15,16]$ and that ICS use leads to delayed virus clearance [17]. Other studies, however, have shown normal responses in patients on ICS [18]. It is important to note that most studies have been carried out with rhinovirus and there may be differences in the response to other viruses.

Conversely, there is evidence to suggest that taking ICS may be beneficial in dealing with virus infections, specifically those due to coronavirus. Pre-treatment of human respiratory epithelial cells in vitro with budesonide, in combination with glycopyrronium and formoterol, has inhibitory actions on coronavirus HCoV-229E replication and cytokine production [19]. Furthermore, early, not yet peer-reviewed data, suggest ciclesonide blocks SARS-CoV-2 RNA replication in vitro [20] and inhibits SARS-CoV-2 cytopathic activity [21], which may be of great relevance to reducing the risk of developing of COVID-19 in response to SARS-CoV-2 infection or reducing the severity of the disease.

ICS use undoubtedly reduces the rate of exacerbations in both asthma and COPD. If people with stable asthma stop or reduce their ICS inappropriately in response to concerns about immunosuppression and worries about developing COVID-19, they may be at significant risk of having an exacerbation. Approximately $40-60 \%$ of COPD and up to $80 \%$ of asthma exacerbations are due to viral infections, including common coronaviruses [22]. Therefore, the use of ICS must either reduce the risk of becoming infected or modify the subsequent inflammatory response and lung damage. In vitro, corticosteroids inhibit rhinovirus and respiratory syncytial virus-induced cytokine release [3] but the timing of exposure to ICS appears important with pre-treatment less effective than administration at the time of infection [23].

Self-management plans advise asthma patients to increase the dose of ICS at the onset of an exacerbation to reduce the risk of progression to a severe event and reduce the need for oral corticosteroids. In people with COVID-19, corticosteroids may theoretically modulate the inflammatory response and reduce the risk of developing ARDS. Systemic corticosteroids were widely used during previous coronavirus outbreaks 
(SARS and Middle East respiratory syndrome (MERS)); however, a systematic review of published literature on their use in SARS was not able to conclude that treatment was beneficial and there was some evidence of possible harm [24]. Current interim guidance from World Health Organization on clinical management of COVID-19 advises against the use of systemic corticosteroids outside of clinical trials [25]. There is currently very low-quality evidence from a case series report from Japan of improvement in three patients with COVID-19 requiring oxygen, but not ventilatory support, after being given inhaled ciclesonide [26]. There was no control group and it is not known whether the patients would have improved spontaneously.

We undertook a rapid systematic review to evaluate whether pre-morbid use or continued administration of inhaled steroids is a risk factor for adverse outcomes in acute respiratory infections due to COVID-19, SARS or MERS. The review was conducted using the PRISMA (Preferred Reporting Items for Systematic Reviews and Meta-Analyses) checklist in March 2020 [27]. We searched PubMed, EMBASE, Scopus and Google Scholar for relevant studies on (COVID-19 or SARS or MERS or coronavirus) and "corticosteroids". As it was not anticipated that there would be any randomised controlled clinical trials available for analysis, case-control, cohort studies and case series were eligible for inclusion provided data from a comparison group was available.

In total 771 publications were identified by the search strategies. The abstracts of these publications were screened by two reviewers (D.M.G. Halpin and D. Singh) and 59 publications were identified for full text review. Following examination of the full texts including translations of those in Chinese, no publications were identified as having data on prior ICS use in patients with SARS, MERS or COVID-19 infection. No data were available for either a qualitative or narrative answer to the review question.

A few studies did report the prevalence of comorbidities, including chronic respiratory disease and occasionally specifically asthma or COPD, but in general studies provided little or no information about patients' pre-morbid health or medication. Even if data on the prevalence of ICS use had been available, their interpretation would have been impossible without also having data on the severity of the underlying respiratory disease to allow adjustment for this as a confounding factor.

At present, there is no evidence as to whether pre-morbid use or continued administration of ICS is a factor for adverse or beneficial outcomes in acute respiratory infections due to coronavirus. Further data and studies are urgently needed. As a start, it is essential that epidemiological studies of COVID-19 include detailed information on patients' comorbidities and prior medication in order to allow analyses of possible benefits or harms of ICS or other therapy. Randomised controlled intervention studies are also needed to assess the benefits of ICS in treating COVID-19 in both patients with and without chronic respiratory disease. These have begun $[28,29]$.

At the present time in this COVID-19 pandemic, clinicians should be aware that there is no evidence to support the withdrawal of ICS in patients treated with these drugs, and to do so is likely to be harmful. Patients with asthma and COPD who are stable while using ICS should continue on their treatment. If there is uncertainty about the diagnosis, physicians should be more careful about initiating ICS or ICS/long-acting $\beta$-agonist in patients without clear objective evidence of asthma. Similarly, there is no evidence to suggest a change in the advice for asthma patients to increase the dose of ICS at the onset of an exacerbation.

Acknowledgements: We wish to thank Rebecca Decker, Executive Director, Global Initiative for Chronic Obstructive Lung Disease (GOLD) for her support and Wei-jie Guan and Ying Zhong for their help with the papers written in Chinese.

Conflict of interest: D. Singh reports grants and personal fees from AstraZeneca, Boehringer Ingelheim, Chiesi, Glenmark, Menarini, Mundipharma, Novartis, Pfizer, Pulmatrix, Theravance and Verona, personal fees from Cipla, Genentech, GlaxoSmithKline, Peptinnovate and Teva, outside the submitted work. R.M. Hadfield has nothing to disclose. D.M.G. Halpin reports personal fees from AstraZeneca, Chiesi, GlaxoSmithKline, Novartis, Pfizer and Sanofi, personal fees and non-financial support from Novartis and Boehringer Ingelheim, outside the submitted work.

Support statement: Financial support for literature searching and obtaining some of the full text papers was provided by the Global Initiative for Chronic Obstructive Lung Disease (GOLD). The authors were not funded to undertake this review.

\section{References}

1 Wu Z, McGoogan JM. Characteristics of and important lessons from the coronavirus disease 2019 (COVID-19) outbreak in China: summary of a report of 72314 cases from the Chinese Center for Disease Control and Prevention. JAMA 2020; in press [https://doi.org/10.1001/jama.2020.2648].

2 Huang C, Wang Y, Li X, et al. Clinical features of patients infected with 2019 novel coronavirus in Wuhan, China. Lancet 2020; 395: 497-506.

3 Oliver BG, Robinson P, Peters M, et al. Viral infections and asthma: an inflammatory interface? Eur Respir J 2014; 44: 1666-1681.

4 Lewis TC, Henderson TA, Carpenter AR, et al. Nasal cytokine responses to natural colds in asthmatic children. Clin Exp Allergy 2012; 42: 1734-1744. 
5 Wark PA, Grissell T, Davies B, et al. Diversity in the bronchial epithelial cell response to infection with different rhinovirus strains. Respirology 2009; 14: 180-186.

6 Halpin DMG, Faner R, Sibila O, et al. Do chronic respiratory diseases or their treatment affect the risk of SARS-CoV-2 infection? Lancet Respir Med 2020; in press [https://doi.org/10.1016/S2213-2600(20)30167-3].

7 The Novel Coronavirus Pneumonia Emergency Response Epidemiology Team. The epidemiological characteristics of an outbreak of 2019 novel coronavirus diseases (COVID-19) - China, 2020. China CDC Weekly 2020; 2: 113-122.

8 Global Initiative for Asthma. Global strategy for asthma management and prevention. 2019. www.ginasthma.org

9 Global Initiative for Chronic Obstructive Lung Disease (GOLD). Global strategy for the diagnosis, management, and prevention of chronic obstructive pulmonary disease. 2020. www.goldcopd.org/

10 Yang $\mathrm{M}$, Zhang $\mathrm{Y}$, Chen $\mathrm{H}$, et al. Inhaled corticosteroids and risk of upper respiratory tract infection in patients with asthma: a meta-analysis. Infection 2019; 47: 377-385.

11 Yang $\mathrm{M}$, Chen $\mathrm{H}$, Zhang $\mathrm{Y}$, et al. Long-term use of inhaled corticosteroids and risk of upper respiratory tract infection in chronic obstructive pulmonary disease: a meta-analysis. Inhal Toxicol 2017; 29: 219-226.

12 Yang IA, Clarke MS, Sim EH, et al. Inhaled corticosteroids for stable chronic obstructive pulmonary disease. Cochrane Database Syst Rev 2012; 7: CD002991.

13 Contoli M, Pauletti A, Rossi MR, et al. Long-term effects of inhaled corticosteroids on sputum bacterial and viral loads in COPD. Eur Respir J 2017; 50: 1700451.

14 McKeever T, Harrison TW, Hubbard R, et al. Inhaled corticosteroids and the risk of pneumonia in people with asthma: a case-control study. Chest 2013; 144: 1788-1794.

15 Davies JM, Carroll ML, Li H, et al. Budesonide and formoterol reduce early innate anti-viral immune responses in vitro. PLoS One 2011; 6: e27898.

16 Simpson JL, Carroll M, Yang IA, et al. Reduced antiviral interferon production in poorly controlled asthma is associated with neutrophilic inflammation and high-dose inhaled corticosteroids. Chest 2016; 149: 704-713.

17 Singanayagam A, Glanville N, Girkin JL, et al. Corticosteroid suppression of antiviral immunity increases bacterial loads and mucus production in COPD exacerbations. Nat Commun 2018; 9: 2229.

18 Southworth T, Pattwell C, Khan N, et al. Increased type 2 inflammation post rhinovirus infection in patients with moderate asthma. Cytokine 2020; 125: 154857.

19 Yamaya M, Nishimura H, Deng X, et al. Inhibitory effects of glycopyrronium, formoterol, and budesonide on coronavirus $\mathrm{HCoV}-229 \mathrm{E}$ replication and cytokine production by primary cultures of human nasal and tracheal epithelial cells. Respir Investig 2020; in press [https://doi.org/10.1016/j.resinv.2019.12.005].

20 Matsuyama S, Kawase M, Nao N, et al. The inhaled corticosteroid ciclesonide blocks coronavirus RNA replication by targeting viral NSP15. bioRxiv 2020; preprint [https://doi.org/10.1101/2020.03.11.987016].

21 Jeon S, Ko M, Lee J, et al. Identification of antiviral drug candidates against SARS-CoV-2 from FDA-approved drugs. bioRxiv 2020; preprint [https://doi.org/10.1101/2020.03.20.999730].

22 Johnston SL. Overview of virus-induced airway disease. Proc Am Thorac Soc 2005; 2: 150-156.

23 Bochkov YA, Busse WW, Brockman-Schneider RA, et al. Budesonide and formoterol effects on rhinovirus replication and epithelial cell cytokine responses. Respir Res 2013; 14: 98.

24 Stockman LJ, Bellamy R, Garner P. SARS: systematic review of treatment effects. PLoS Med 2006; 3: e343.

25 World Health Organization. Clinical management of severe acute respiratory infection (SARI) when COVID-19 disease is suspected. Interim guidance. www.who.int/publications-detail/clinical-management-of-severe-acuterespiratory-infection-when-novel-coronavirus-. Date last updated: 13 March 2020; date last accessed: 15 April 2020.

26 Iwabuchi K, Yoshie K, Kurakami Y, et al. [COVID-19 Three cases improved with inhaled ciclesonide in the early to middle stages of pneumonia]. www.kansensho.or.jp/uploads/files/topics/2019ncov/covid19_casereport_200310. pdf Date last updated: 9 March 2020; date last accessed: 15 April 2020

27 Moher D, Liberati A, Tetzlaff J, et al. Preferred reporting items for systematic reviews and meta-analyses: the PRISMA statement. PLoS Med 2009; 6: e1000097.

28 A trial of ciclesonide in adults with mild COVID-19. NCT04330586. https://clinicaltrials.gov/ct2/show/ NCT04330586 Date last updated: 1 April 2020; date last accessed: 15 April 2020.

29 Protective Role of Inhaled Steroids for Covid-19 Infection (INHASCO). NCT04331054. https://clinicaltrials.gov/ ct2/show/NCT04331054 Date last updated: 17 April 2020; date last accessed: 15 April 2020. 